\title{
Well-posedness of first order semilinear PDEs by stochastic perturbation
}

\author{
Christian Olivera 1 \\ Departamento de Matemática, Universidade Estadual de Campinas, \\ F. 54(19) 3521-5921? Fax 54(19) 3521-6094 \\ 13.081-970 - Campinas - SP, Brazil. ; colivera@ime.unicamp.br
}

\begin{abstract}
We show that first order semilinear PDEs by stochastic perturbation are well-posedness for globally Holder continuous and bounded vector field, with an integrability condition on the divergence. This result extends the liner case presented in [2]. The proof is based on in the stochastic characteristics method and a version of the commuting Lemma.
\end{abstract}

Key words: Stochastic characteristic method, First order stochastic partial differential equations, Stochastic perturbation, commuting Lemma.

MSC2000 subject classification: $60 \mathrm{H} 10,60 \mathrm{H} 15$.

\section{Introduction}

This work is motivated by the paper 2] where the linear equation

$$
\left\{\begin{array}{l}
d u(t, x)+b(t, x) \nabla u(t, x) d t+\nabla u(t, x) \circ d B_{t}=0 \\
u(0, x)=f(x) \in L^{\infty}\left(\mathbb{R}^{d}\right)
\end{array}\right.
$$

\footnotetext{
${ }^{1}$ Research partially supported by FAPESP 2012/18739-0.
} 
has been studied, was proved existence and uniqueness of $L^{\infty}$ - solutions for a globally Holder continuous and bounded vector field, with an integrability condition on the divergence, and where $B_{t}=\left(B_{t}^{1}, \ldots, B_{t}^{d}\right)$ is a standard Brownian motion in $\mathbb{R}^{d}$.

The aim of this paper is to investigate parts of this theory under the effect of nonlinear terms. Namely, we considerer the semilinear SPDE

$$
\left\{\begin{array}{l}
d u(t, x)+b(t, x) \nabla u(t, x) d t+F(t, x, u) d t+\nabla u(t, x) \circ d B_{t}=0 \\
u(0, x)=f(x) \in L^{\infty}\left(\mathbb{R}^{d}\right) .
\end{array}\right.
$$

We shall prove the existence and uniqueness of weak $L^{\infty}$ - solutions for a globally Holder continuous and bounded vector field, with an integrability condition on the divergence. Moreover, we obtain a representation of the solution via stochastic flows. This is a example of nonlinear SPDE where the stochastic perturbation makes the equation well-posedness.

The fundamental tools used here is the stochastic characteristics method (see for example [1], [5] and [7] ) and the version of the commuting Lemma presented in [2]. That is, we follows the strategy given in [2] in combination with the stochastic characteristics method.

The article is organized as follows: Section 2 we shall define the concept of weak $L^{\infty}$-solutions for the equation (2) and we shall prove existence for this class of solutions. . In section 3, we shall show a uniqueness theorem for weak $L^{\infty}$-solutions.

Through of this paper we fix a stochastic basis with a $d$-dimensional Brownian motion $\left(\Omega, \mathcal{F},\left\{\mathcal{F}_{t}: t \in[0, T]\right\}, \mathbb{P},\left(B_{t}\right)\right)$.

\section{$2 \quad$ Existence of weak $L^{\infty}$-solutions}

Let $T>0$ be fixed. For $\alpha \in(0,1)$ define the space $L^{\infty}\left([0, T], C^{\alpha}\left(\mathbb{R}^{d}\right)\right)$ as the set of all bounded Borel functions $f:[0, T] \times \mathbb{R}^{d} \rightarrow \mathbb{R}$ for which 


$$
[f]_{\alpha, T}=\sup _{t \in[0, T]} \sup _{x \neq y \in \mathbb{R}^{d}} \frac{|f(x)-f(y)|}{|x-y|}
$$

We write the $L^{\infty}\left([0, T], C^{\alpha}\left(\mathbb{R}^{d}, \mathbb{R}^{d}\right)\right)$ for the space of all vector fields having components in $L^{\infty}\left([0, T], C^{\alpha}\left(\mathbb{R}^{d}\right)\right)$.

We shall assume that

$$
\begin{gathered}
b \in L^{\infty}\left([0, T], C^{\alpha}\left(\mathbb{R}^{d}, \mathbb{R}^{d}\right)\right), \\
\text { Div } b \in L^{p}\left([0, T] \times \mathbb{R}^{d}\right) \text { for } p>2 . \\
F \in L^{1}\left([0, T], L^{\infty}\left(\mathbb{R}^{d} \times \mathbb{R}\right)\right)
\end{gathered}
$$

and

$$
F \in L^{\infty}\left([0, T] \times \mathbb{R}^{d}, L I P(\mathbb{R})\right) .
$$

\subsection{Definition of weak $L^{\infty}$-solutions}

Definition 2.1 We assume (3), (4), (5) and (6)). A weak $L^{\infty}$-solution of the Cauchy problem (2) is a stochastic process $u \in L^{\infty}\left(\Omega \times[0, T] \times \mathbb{R}^{d}\right)$ such that, for every test function $\varphi \in C_{0}^{\infty}\left(\mathbb{R}^{d}\right)$, the process $\int u(t, x) \varphi(x) d x$ has a continuous modification which is a $\mathcal{F}_{t}$-semimartingale and satisfies

$$
\begin{gathered}
\int u(t, x) \varphi(x) d x=\int f(x) \varphi(x) d x \\
+\int_{0}^{t} \int b(s, x) \nabla \varphi(x) u(s, x) d x d s+\int_{0}^{t} \int \operatorname{div} b(s, x) \varphi(x) u(s, x) d x d s \\
+\int_{0}^{t} \int F(s, x, u) \varphi(x) d x d s+\sum_{i=0}^{d} \int_{0}^{t} \int D_{i} \varphi(x) u(s, x) d x \circ d B_{s}^{i}
\end{gathered}
$$


Remark 2.1 We observe that a weak $L^{\infty}$-solution in the previous Stratonovich sense satisfies the Itô equation

$$
\begin{gathered}
\int u(t, x) \varphi(x) d x=\int f(x) \varphi(x) d x \\
+\int_{0}^{t} \int b(s, x) \nabla \varphi(x) u(s, x) d x d s+\int_{0}^{t} \int \operatorname{div} b(s, x) \varphi(x) u(s, x) d x d s \\
+\int_{0}^{t} \int F(s, x, u) \varphi(x) d x d s+\sum_{i=0}^{d} \int_{0}^{t} \int D_{i} \varphi(x) u(s, x) d x d B_{s}^{i}+\frac{1}{2} \int_{0}^{t} u(s, x) \triangle \varphi(x) d x d s
\end{gathered}
$$

for every test function $\varphi \in C_{0}^{\infty}\left(\mathbb{R}^{d}\right)$. The converse is also true.

\section{$2.2 \quad$ Existence of weak $L^{\infty}$-solutions}

Lemma 2.1 Let $f \in L^{\infty}\left(\mathbb{R}^{d}\right)$. We assume (3), (4), (5) and (6). Then there exits a weak $L^{\infty}$-solution $u$ of the SPDE (2).

Proof: Step 1 Assume that $F \in L^{1}\left([0, T], C_{b}^{\infty}\left(\mathbb{R}^{d} \times \mathbb{R}\right)\right)$ and $f \in C_{b}^{\infty}\left(\mathbb{R}^{d}\right)$. We take a mollifier regularization $b_{n}$ of $b$. It is known (see [1], chapter 1 ) that there exist an unique classical solution $u_{n}(t, x)$ of the SPDE (2), that written in weak Itô form is (7) with $b_{n}$ in place of $b$. Moreover,

$$
u_{n}(t, x)=Z_{t}^{n}\left(x, f\left(Y_{t}^{n}\right)\right)
$$

where $Y_{t}^{n}$ is the inverse of $X_{t}^{n}, X_{t}^{n}(x)$ and $Z_{t}^{n}(x, r)$ satisfy the following equations

$$
X_{t}^{n}=x+\int_{0}^{t} b_{n}\left(s, X_{s}^{n}\right) d s+B_{t}
$$

and

$$
Z_{t}^{n}=r+\int_{0}^{t} F\left(s, X_{s}^{n}(x), Z_{s}^{n}\right) d s
$$


According to theorem 5 of [2], see too remark 8, we have that

$$
\lim _{n \rightarrow \infty} \mathbb{E}\left[\int_{K} \sup _{t \in[0, T]}\left|X_{t}^{n}-X_{t}\right| d x\right]=0
$$

and

$$
\lim _{n \rightarrow \infty} \mathbb{E}\left[\int_{K} \sup _{t \in[0, T]}\left|D X_{t}^{n}-D X_{t}\right| d x\right]=0
$$

for any compact set $K \subset \mathbb{R}^{d}$, where $X_{t}(x)$ verifies

$$
X_{t}=x+\int_{0}^{t} b\left(s, X_{s}\right) d s+B_{t}
$$

Now, we denote

$$
u(t, x)=Z_{t}\left(x, f\left(Y_{t}\right)\right)
$$

$Y_{t}$ is the inverse of $X_{t}$,

and

$$
Z_{t}=r+\int_{0}^{t} F\left(s, X_{s}(x), Z_{s}\right) d s .
$$

Then, we observe that

$$
\begin{gathered}
\left|u^{n}(t, x)-u(t, x)\right| \leq\left|f\left(Y_{t}\right)-f\left(Y_{t}^{n}\right)\right|+\int_{0}^{t} \mid F\left(s, X_{s}^{n}, Z_{s}^{n}\left(f\left(Y_{t}^{n}\right)\right)-F\left(s, X_{s}, Z_{s}\left(f\left(Y_{t}\right)\right) \mid d s\right.\right. \\
\leq\left|f\left(Y_{t}\right)-f\left(Y_{t}^{n}\right)\right|+C \int_{0}^{t}\left|Z_{s}^{n}\left(f\left(Y_{t}^{n}\right)\right)-Z_{s}\left(f\left(Y_{t}\right)\right)\right| d s .
\end{gathered}
$$

From to theorem 5 of [2], see too remark 8, and the Lipchitz property of $F$ we conclude that $\lim _{n \rightarrow \infty} \mathbb{E}\left[\int_{K} \sup _{t \in[0, T]}\left|u_{n}(t, x)-u(t, x)\right|\right]=0$ and $u(t, x)$ is a weak $L^{\infty}$-solution of the $\operatorname{SPDE}(2)$.

Step 2 Assume that $F \in L^{1}\left([0, T], C_{b}^{\infty}\left(\mathbb{R}^{d} \times \mathbb{R}\right)\right)$. We a take a mollifier regularization $f_{n}$ of $f$. By the last step $u_{n}(t, x)=Z_{t}\left(x, f_{n}\left(Y_{t}\right)\right)$ is a weak 
$L^{\infty}$-solution of the SPDE (2), that written in weak Itô form is (7) with $f_{n}$ in place of $f$.

We have that any compact set $K \subset \mathbb{R}^{d}$ and $p \geq 1$

$$
\begin{gathered}
\lim _{n \rightarrow \infty} \sup _{[0, T]} \int_{K}\left|f_{n}\left(X_{t}^{-1}\right)-f\left(X_{t}^{-1}\right)\right|^{p} d x= \\
\lim _{n \rightarrow \infty} \sup _{[0, T]} \int_{X_{t}(K)}\left|f_{n}(x)-f(x)\right|^{p} J X_{t}(x) d x=0
\end{gathered}
$$

Then we have that

$$
\lim _{n \rightarrow \infty} \sup _{[0, T]} \int_{K}\left|Z_{t}\left(x, f_{n}\left(Y_{t}\right)\right)-Z_{t}\left(x, f\left(Y_{t}\right)\right)\right|^{p} d x=0 .
$$

Thus $u(t, x)=Z_{t}\left(x, f\left(Y_{t}\right)\right)$ is a weak $L^{\infty}$-solution of the SPDE (2)

Step 3 We take a mollifier regularization $F_{n}$ of $F$. By the step 2, we have that $u_{n}(t, x)=Z_{t}^{n}\left(x, f\left(Y_{t}\right)\right)$ is a weak $L^{\infty}$-solution of the SPDE (2), and hold that $Z_{t}^{n}(x, r)$ satisfies the equation (11) with $F_{n}$ in place of $F$. We observe that

$$
\begin{gathered}
\left|Z_{t}^{n}(x, r)-Z_{t}(x, r)\right| \leq \int_{0}^{t}\left|F_{n}\left(t, X_{s}, Z_{s}^{n}\right)-F\left(t, X_{s}, Z_{s}\right)\right| d s \\
\leq \int_{0}^{t}\left|F_{n}\left(t, X_{s}, Z_{s}^{n}\right)-F_{n}\left(t, X_{s}, Z_{s}\right)\right| d s+\int_{0}^{t}\left|F_{n}\left(t, X_{s}, Z_{s}\right)-F\left(t, X_{s}, Z_{s}\right)\right| d s \\
\leq C \int_{0}^{t}\left|Z_{s}^{n}-Z_{s}\right| d s+\int_{0}^{t}\left|F_{n}\left(t, X_{s}, Z_{s}\right)-F\left(t, X_{s}, Z_{s}\right)\right| d s
\end{gathered}
$$

By the Gronwall Lemma we follow that

$$
\lim _{n \rightarrow \infty}\left|Z_{t}^{n}(x, r)-Z_{t}(x, r)\right|=0 \text { uniformaly in } t, x, r .
$$

Then

$$
\lim _{n \rightarrow \infty} \mid Z_{t}^{n}\left(x, f\left(Y_{t}\right)\right)-Z_{t}\left(x, f\left(Y_{t}\right)\right)=0 \text { uniformaly in } t \text { and } x .
$$

Therefore, we conclude that $u(t, x)=Z_{t}\left(x, f\left(Y_{t}\right)\right)$ is a weak $L^{\infty}$ - solution of the SPDE (2). 


\section{$3 \quad$ Uniqueness of weak $L^{\infty}$-solutions}

In this section, we shall present an uniqueness theorem for the SPDE (2) under similar conditions to the linear case, see theorem 20 of [2].

Let $\varphi_{n}$ be a standard mollifier. We introduced the commutator defined as

$$
\mathcal{R}_{n}(b, u)=(b \nabla)\left(\varphi_{n} * u\right)-\varphi_{n} *((b \nabla) u)
$$

We recall here the following version of the commutator lemma which is at the base of our uniqueness theorem.

Lemma 3.1 Let $\phi_{t}$ be an $C^{1}$-diffeomorphism of $\mathbb{R}^{d}$. Assume $b \in L_{\text {loc }}^{\infty}\left(\mathbb{R}^{d}, \mathbb{R}^{d}\right)$ , divb $\in L_{\text {loc }}^{1}\left(\mathbb{R}^{d}\right), u \in L_{\text {loc }}^{\infty}\left(\mathbb{R}^{d}\right)$. Moreover, for $d>1$, assume also $\mathrm{J}^{-1} \in$ $W_{\text {loc }}^{1,1}\left(\mathbb{R}^{d}\right)$ Then for any $\rho \in C_{0}^{\infty}\left(\mathbb{R}^{d}\right)$ there exits a constant $C_{\rho}$ such that , given any $R>0$ such that $\operatorname{supp}\left(\rho \circ \phi^{-1}\right) \subset B(R)$, we have :

a) for $d>1$

$$
\begin{gathered}
\left|\int \mathcal{R}_{n}(b, u)(\phi(x)) \rho(x) d x\right| \\
\leq C_{\rho}\|u\|_{L_{R+1}^{\infty}}\left[\|d i v b\|_{L_{R+1}^{1}}\left\|J \phi^{-1}\right\|_{L_{R}^{\infty}}+\|b\|_{L_{R+1}^{\infty}}\left(\left\|D \phi^{-1}\right\|_{L_{R}^{\infty}}+\left\|D J \phi^{-1}\right\|_{L_{R}^{1}}\right)\right]
\end{gathered}
$$

b) for $d=1$

$$
\left|\int \mathcal{R}_{n}(b, u)(\phi(x)) \rho(x) d x\right| \leq C_{\rho}\|u\|_{L_{R+2}^{\infty}}\|b\|_{W_{R+2}^{1,1}}\left\|J \phi^{-1}\right\|_{L_{R}^{\infty}}
$$

Proof: See pp 28 of [2].

We are ready to prove our uniqueness result of weak $L^{\infty}$-solution to the Cauchy problem (2).

Theorem 3.1 Assume (3), (4), (5) and (6). Then, for every $f \in L^{\infty}\left(\mathbb{R}^{d}\right)$ there exists an unique weak $L^{\infty}$-solution of the Cauchy problem (2). 


\section{Proof:}

Step 1( Itô-Ventzel-Kunita formula) Let $u, v$ be are two weak $L^{\infty}$-solutions and $\varphi_{n}$ be a standard mollifier. We put $w=u-v$, applying the Itô-VentzelKunita formula (see Theorem 8.3 of [6] ) to $F(y)=\int w(t, z) \varphi_{n}(y-z) d z$, we obtain that

$$
\int w(t, z) \varphi_{n}\left(X_{s}-z\right) d z
$$

is equal to

$$
\begin{aligned}
& \int_{0}^{t} \int b(s, z) \nabla\left[\varphi_{n}\left(X_{s}-z\right)\right] w(s, z) d z d s+\int_{0}^{t} \int \operatorname{div} b(s, z) \varphi_{n}\left(X_{s}-z\right) u(s, z) d z d s+ \\
& \left.\int_{0}^{t} \int(F(s, z, u)-F(s, z, v)) \varphi_{n}\left(X_{s}-z\right) d z d s+\sum_{i=1}^{d} \int_{0}^{t} \int w(s, z) D_{i}\left[\varphi_{n}\left(X_{s}-z\right)\right]\right] d z \circ d B_{s}^{i}+ \\
& \int_{0}^{t} \int(b \nabla)\left(w(s, .) * \varphi_{n}\right)\left(X_{s}\right) d s-\sum_{i=1}^{d} \int_{0}^{t} \int w(s, z) D_{i}\left[\varphi_{n}\left(X_{s}-z\right)\right] d z \circ d B_{s}^{i} .
\end{aligned}
$$

Then

$$
\begin{gathered}
\int w(t, z) \varphi_{n}\left(X_{t}-z\right) d z= \\
\int_{0}^{t} \int(F(s, z, u)-F(s, z, v)) \varphi_{n}\left(X_{s}-z\right) d z d s-\int_{0}^{t} \mathcal{R}_{n}(w, b)\left(X_{s}(x)\right) d s
\end{gathered}
$$

where $\mathcal{R}_{n}$ is the commutator defined above.

Step $2\left(\lim _{n \rightarrow \infty} \int_{0}^{t} \mathcal{R}_{n}(w, b)\left(X_{s}\right) d s=0\right)$ We argue as in [2]. We observe by Lemma 3.1 and the Lebesgue dominated theorem that

$$
\lim _{n \rightarrow \infty} \int_{0}^{t} \int \mathcal{R}_{n}(w, b)\left(X_{s}\right) \rho(x) d s=0
$$

for all $\rho \in C_{0}^{\infty}\left(\mathbb{R}^{d}\right)$, for details see Theorem 20 of [2].

Step 3( $w=0)$ We observe that 


$$
\lim _{n \rightarrow \infty}\left(w(t, .) * \varphi_{n}\right)(.)=w(t, .)
$$

, where the convergence is in $L^{1}\left([0, T], L_{l o c}^{1}\left(\mathbb{R}^{d}\right)\right)$. From the flow properties of $X_{t}$, see theorem 5 of [2], we obtain

$$
\lim _{n \rightarrow \infty}\left(w(t, .) * \varphi_{n}\right)\left(X_{t}\right)=w\left(t, X_{t}\right)
$$

and

$$
\begin{gathered}
\lim _{n \rightarrow \infty}\left((F(t, ., u)-F(t, ., v)) * \varphi_{n}\right)\left(X_{t}\right)= \\
\left(F\left(t,, X_{t}, u\left(t,, X_{t}\right)\right)-F\left(t,, X_{t}, v\left(t, X_{t}\right)\right),\right.
\end{gathered}
$$

where the convergence is $\mathbb{P}$ a.s in $L^{1}\left([0, T], L_{l o c}^{1}\left(\mathbb{R}^{d}\right)\right)$. Then by steps 1,2 we have

$$
w\left(t, X_{t}\right)=\int_{0}^{t} F\left(s, X_{s}, u\left(t,, X_{s}\right)\right)-F\left(s, X_{s}, v\left(t, X_{s}\right)\right) d s .
$$

Thus, for any compact set $K \subset \mathbb{R}^{d}$ we obtain that

$$
\begin{gathered}
\int_{K}\left|w\left(t, X_{t}\right)\right| d x \leq \int_{0}^{t} \int_{K}\left|F\left(s,, X_{s}, u\left(t, X_{s}\right)\right)-F\left(s, X_{s}, v\left(t, X_{s}\right)\right)\right| d x d s \\
\leq C \int_{0}^{t} \int_{K}\left|w\left(t,, X_{s}\right)\right| d x d s
\end{gathered}
$$

where $C$ is contant related to the Lipchitz property of $F$. It follows

$$
\int_{K}\left|w\left(t, X_{t}\right)\right| d x \leq C \int_{0}^{t} \int_{K}\left|w\left(t, X_{s}\right)\right| d x d s .
$$

and thus $w\left(t, X_{t}\right)=0$ by the Gronwall Lemma. 
Remark 3.1 We observe that the unique solution $u(t, x)$ has the representation $u(t, x)=Z_{t}\left(x, f\left(X_{t}^{-1}\right)\right)$, where $X_{t}$ and $Z_{t}$ satisfy the equations (10) and (11) respectively.

Remark 3.2 We mention that other variants of the theorem [3.1 can be proved. In fact, the step 2 is valid under other hypotheses, see corollary 23 of [2].

Remark 3.3 We recall that relevant examples of non-uniqueness for the deterministic linear transport equation are presented in [2] and [3]. Currently we do not get a counter-example itself of the non-linear case. An interesting future work is to study if the nonlinear case may induce new pathologies.

\section{References}

[1] Chow, P. L. 2007. Stochastic Partial Differential Equations, Chapman Hall/CRC.

[2] Flandoli F., Gubinelli M. , Priola, E. 2010. Well-posedness of the transport equation by stochastic perturbation, Invent. Math., 180 (1): $1-53$.

[3] Flandoli,F. 2011. The Interaction Between Noise and Transport Mechanisms in PDEs, Milan j. Math, 79 (2) : 543-560.

[4] Flandoli, F. 2009. Remarks on uniqueness and strong solutions to deterministic and stochastic differential equations, Metrika, 69 (2-3) : 101-123.

[5] Kunita, H. 1984. First Order Stochastic Partial Differential Equations, in Proceedings of the Taniguchi International Symposium on Stochastic Analysis, North-Holland Mathematical Library, 249-269. 
[6] Kunita, H. 1982. Stochastic differential equations and stochastic flows of diffeomorphisms, Lectures Notes in Mathematics, 1097 : 143-303.

[7] Kunita, H. 1990. Stochastic flows and stochastic differential equations, Cambridge University Press . 